\title{
REMARKS ON DILWORTH'S THEOREM IN RELATION TO TRANSVERSAL THEORY
}

\author{
by HAZEL PERFECT
}

(Received 10 August, 1978)

In his book Transversal Theory [3], L. Mirsky has remarked that "At present, $\dagger$ the relation between Dilworth's decomposition theorem ... and transversal theory is rather tenuous; but further study may reveal unexpected connections". Some of these connections can perhaps now be seen a little more clearly; and our purpose in this note is to make one or two observations in this regard. Throughout, all sets considered are finite.

The theorem of Dilworth to which reference is made was first proved in [1]. Simpler proofs due to Perles [6], Tverberg [7] and F. Galvin (personal communication to Professor Mirsky) are now available. It appears, however, that in all probability only a restricted form of Dilworth's theorem is of special relevance to transversal theory; namely when the underlying partially ordered set contains no chain of length greater than 2 . We begin, therefore, with a very simple proof of this special case (which owes something to Perles's general proof).

A RESTRICTED FORM OF DILWORTH'S THEOREM. Let $(X,<)$ be a partially ordered set in which no chain has length greater than 2 . Further, let the maximum length of an antichain in $(X,<)$ be $m$. Then $X$ can be covered by $m$ chains.

Proof. We may suppose that $|X|>m>1$, since otherwise the result is obvious. We proceed by induction on $m$ and assume that the theorem is true for partially ordered sets in which the maximum length of an antichain is less than $\mathrm{m}$.

Denote by $X^{+}$(respectively $X^{-}$) the set of maximal (respectively minimal) elements of $X$. Then $X=X^{+} \cup X^{-}$. (It is not, of course, assumed that $X^{+} \cap X^{-}=\varnothing$.)

Case 1. There is an antichain $A$ in $X$ with $|A|=m, A \nsubseteq X^{+}, A \nsubseteq X^{-}$.

Let us write

$$
A=\left\{a_{1}^{+}, \ldots, a_{s}^{+}, a_{s+1}^{-}, \ldots, a_{m}^{-}\right\},
$$

where $a_{i}^{+} \in X^{+}(1 \leq i \leq s), a_{i}^{-} \notin X^{+}(s+1 \leq i \leq m)$. Then $0<s<m$. Write

$$
X_{1}=\left\{a_{1}^{+}, \ldots, a_{s}^{+}\right\} \cup\left\{x \in X^{-}: x \neq a_{s+1}^{-}, \ldots, x \neq a_{m}^{-}\right\}
$$

and

$$
X_{2}=\left\{a_{s+1}^{-}, \ldots, a_{m}^{-}\right\} \cup\left\{y \in X^{+}: y \neq a_{1}^{+}, \ldots, y \neq a_{s}^{+}\right\} .
$$

Then $X=X_{1} \cup X_{2}$. Further, every element of $X_{1}$ is incomparable with each of $a_{s+1}^{-}, \ldots, a_{m}^{-}$, and every element of $X_{2}$ is incomparable with each of $a_{1}^{+}, \ldots, a_{s}^{+}$. Therefore $X_{1}$ (respectively $X_{2}$ ) cannot contain an antichain of more than $s$ (respectively $m-s$ )

† This was in 1971.

Glasgow Math. J. 21 (1980) 19-22. 
elements; and, by the induction hypothesis, $X_{1}$ (respectively $X_{2}$ ) can be covered by $s$ (respectively $m-s$ ) chains. So $X$ can be covered by $m$ chains.

Case 2. The only antichains of $X$ of length $M$ are subsets of $X^{+}$or $X^{-}$.

Since each of $X^{+}$and $X^{-}$is itself an antichain, these are the only possibilities for antichains of length $m$. Without loss of generality, suppose $X^{+}$is an antichain of length $m$. Evidently, then, there must exist $a \in X^{+}, b \in X^{-}$with $a \geq b$. If $X^{\prime}=X \backslash\{a, b\}$, then $X^{\prime}$ can contain no antichain of length $m$; for this would imply that $X$ contained an antichain of length $m$ different from $X^{+}$and $X^{-}$. So, by the induction hypothesis, $X^{\prime}$ can be covered by $m-1$ chains; and hence $X$ can be covered by $m$ chains.

The reader familiar with König's duality theorem [2, p. 233] (see also [3, p. 22]) will readily see that this theorem is virtually equivalent to the above restricted form of Dilworth's theorem. We simply associate a partial order with a bipartite graph in the most obvious way, and observe that minimum separating sets and maximum antichains feature as complementary sets.

In [3, p. 63-64] a deduction of P. Hall's classic theorem from the restricted form of Dilworth's theorem is described. $\dagger$ We support our contention that this restricted form is the all-important one for transversal theory by providing now a very simple deduction from it of a much more general theorem on common transversals. Many proofs of this theorem are now known and we refer the interested reader again to [3]. The proof which follows depends on essentially the same initial construction as that in [4] for the special case when $M=\varnothing$, though there the deduction was from Hall's theorem. A similar deduction from Hall's theorem for the case of general $M$ was briefly indicated in [5].

TheOREM. Let $\mathfrak{A}=\left(A_{i}: i \in I\right), \mathfrak{B}=\left(B_{j}: j \in J\right)$, with $|I|=|J|=n$, be families of subsets of $E$; and let $M \subseteq E$ be given. Then $\mathfrak{A}$ and $\mathfrak{B}$ have a common transversal containing $M$ if and only if

$$
\left|A\left(I^{\prime}\right) \cap B\left(J^{\prime}\right)\right|+\left|\left(A\left(I^{\prime}\right) \cup B\left(J^{\prime}\right)\right) \cap M^{\prime}\right| \geq\left|I^{\prime}\right|+\left|J^{\prime}\right|+\left|M^{\prime}\right|-n, \forall I^{\prime} \subseteq I, J^{\prime} \subseteq J, M^{\prime} \subseteq M .
$$

Remark. More familiar conditions, easily seen to be equivalent to $(*)$, have $M$ in place of $M^{\prime}$. As is usual, we have written, for instance, $A\left(I^{\prime}\right)$ to denote $\bigcup_{i \in I^{\prime}} A_{i}$.

Proof. We shall prove only the sufficiency of the conditions $(*)$, since their necessity is readily checked.

Take sets $E^{1}, E^{2}$ with $\left|E^{1}\right|=\left|E^{2}\right|=|E|$, and $M^{1} \subseteq E^{1}, M^{2} \subseteq E^{2}$ with $\left|M^{1}\right|=\left|M^{2}\right|=|M|$; and suppose $I, J, E^{1}, E^{2}$ are pairwise disjoint. We regard $E^{1}$ and $E^{2}$ as 'copies' of $E$ (more formally, we set up bijections $E \rightarrow E^{1}, E \rightarrow E^{2}$ ) and, for any element $e$ or subset $X$ of $E$, we denote its copy in $E^{i}$ by $e^{i}$ or $X^{i}$ respectively $(i=1,2)$. On the set $I \cup E^{1} \cup E^{2} U$ $J$ we define a partial order in the following way: the comparable elements are specified by the relations

$$
\begin{array}{ccc}
i>e^{1} & \text { whenever } & e \in A_{i}(i \in I), \\
e^{2}>j & \text { whenever } & e \in B_{j}(j \in J), \\
e^{1}<e^{2} & \text { whenever } & e \in E \backslash M .
\end{array}
$$

$\dagger$ Another deduction described in [3], which just verges on transversal theory, is Rado's theorem on representing sets. (See p. 190.) 
Evidently no chain has length greater than 2. Also if $I \cup E^{1} \cup E^{2} \cup J$ can be covered by $|E|+n$ chains (it cannot be covered by fewer), then these chains must consist of $n$ chains which cover $I$ and $X^{1}$ say, where $E^{1} \supseteq X^{1} \supseteq M^{1}$, another $n$ chains which cover $X^{2}\left(\supseteq M^{2}\right)$ and $J$, and another $|E|-n$ chains which cover $E^{1} \backslash X^{1}$ and $E^{2} \backslash X^{2}$. It follows readily that, in this situation, $\mathfrak{A}$ and $\mathfrak{B}$ have a common transversal (namely $X$ ) containing $M$. $\dagger$ This is the crux.

In order to complete the proof, we shall show that the conditions (*) imply that every antichain in the partially ordered set has length at most $|E|+n$. Let, then, $\tilde{I} \cup \tilde{E}^{1} \cup \tilde{M}^{1} \cup$ $\tilde{E}^{2} \cup \tilde{M}^{2} \cup \tilde{J}$, with $\tilde{I} \subseteq I, \tilde{E}^{1} \subseteq E^{1} \backslash M^{1}, \tilde{M}^{1} \subseteq M^{1}, \tilde{E}^{2} \subseteq E^{2} \backslash M^{2}, \tilde{M}^{2} \subseteq M^{2}, \tilde{J} \subseteq J$, be an antichain. Then

$$
\begin{gathered}
A(\tilde{I})^{1} \cap\left(\tilde{M}^{1} \cup \tilde{E}^{1}\right)=\varnothing, \quad B(\tilde{J})^{2} \cap\left(\tilde{M}^{2} \cup \tilde{\tilde{E}}^{2}\right)=\varnothing, \\
\tilde{E}^{1} \cap \widetilde{E}^{1}=\varnothing .
\end{gathered}
$$

Therefore, in $E$, we have

$$
\begin{gathered}
A(\tilde{I}) \cap(\tilde{M} \cup \tilde{E})=\varnothing, \quad B(\tilde{J}) \cap(\tilde{M} \cup \tilde{E})=\varnothing, \\
\tilde{E} \cap \tilde{E}=\varnothing ;
\end{gathered}
$$

and so, by $(*)$,

$$
\begin{aligned}
|\tilde{I}|+ & \left|\tilde{E}^{1}\right|+\left|\tilde{M}^{1}\right|+\left|\widetilde{E}^{2}\right|+\left|\tilde{M}^{2}\right|+|\tilde{J}| \\
& =|\tilde{I}|+|\tilde{E}|+|\tilde{M}|+|\tilde{E}|+|\tilde{M}|+|\tilde{J}| \\
& \leq|A(\tilde{I}) \cap B(\tilde{I})|+|(A(\tilde{I}) \cup B(\tilde{J})) \cap \tilde{M}|+|\tilde{E}|+|\tilde{E}|+|\tilde{M}|+n \\
& \leq|E|+n .
\end{aligned}
$$

A rather similar deduction from König's theorem may obviously be given, in view of our previous remarks. Both deductions avoid the somewhat tedious technicalities of the proof indicated in [5].

It is a pleasure to me to record my indebtedness to Professor Mirsky for his help and advice in the preparation of this note.

\section{REFERENCES}

1. R. P. Dilworth, A decomposition theorem for partially ordered sets, Ann. of Math. (2) 51 (1950), 161-166.

2. D. König, Theorie der endlichen und unendlichen Graphen (Leipzig, 1936).

3. L. Mirsky, Transversal theory (Academic Press, 1971). 66-67.

4. H. Perfect, Remark on a criterion for common transversals, Glasgow Math. J. 10 (1969),

5. H. Perfect, Marginal elements in transversal theory, Studies in Pure Mathematics (Ed. L. Mirsky, Academic Press, 1971).

$†$ The converse of this statement is also true; but we make no use of this. 
6. M. A. Perles, A proof of Dilworth's decomposition theorem for partially ordered sets, Israel J. Math. 1 (1963), 105-107.

7. H. Tverberg, On Dilworth's decomposition theorem for partially ordered sets, J. Combinational Theory 3 (1967), 305-306.

UNIVERSITY OF SHEFFIELD 